\title{
Effects of Low Temperature and Wind Treatment on Physiological Indexes, Rumen Microbiota, Immune Responses and Hormones in Sheep
}

\section{Hongran Guo}

Northwest Agriculture and Forestry University

\section{Guangchen Zhou}

Northwest Agriculture and Forestry University

Guangjie Tian

Northwest Agriculture and Forestry University

Yuyang Liu

Northwest Agriculture and Forestry University

Ning Dong

Northwest Agriculture and Forestry University

\section{Shijun Zhang}

Northwest Agriculture and Forestry University

Haochen Chai

Northwest Agriculture and Forestry University

Yulin Chen

Northwest Agriculture and Forestry University

Yuxin Yang ( $\nabla$ yangyuxin2002@126.com )

Northwest Agriculture and Forestry University https://orcid.org/0000-0002-1742-2328

\section{Research}

Keywords: cold chill effect, rumen microbiota, antioxidant enzymes, immune response, average daily gain

Posted Date: June 24th, 2020

DOl: https://doi.org/10.21203/rs.3.rs-36618/v1

License: (c) (1) This work is licensed under a Creative Commons Attribution 4.0 International License.

Read Full License 


\section{Abstract}

Background: Low-temperature environments can strongly affect the normal growth and health of livestock. Previous studies have shown that cold exposure can alter the intestinal microbiota and thereby affect other traits. In winter, cold weather can be accompanied by strong winds that aggravate the effects of cold on livestock. In this study, an experiment was conducted to investigate the effect of low temperature and wind speed on physiological indexes, rumen microbiota, and immune responses in sheep.

Methods: The sheep were divided into control group and test group according to their ambient temperature.Sheep in the test group were divided into four groups according to wind-speed treatment: no wind (average wind velocity less than $0.5 \mathrm{~m} / \mathrm{s}$ ), low wind velocity (average wind velocity of $3 \mathrm{~m} / \mathrm{s}$ ), medium wind velocity (average wind velocity of $4 \mathrm{~m} / \mathrm{s}$ ) and high wind velocity (average wind velocity of 5 $\mathrm{m} / \mathrm{s})$.

Results: Average daily gain and the utilization of forage, especially soluble fiber, decreased with increasing wind velocity in cold temperature $(P<0.05)$. In rumen, the enzyme activity of cellulose degradation was also lowerwith increasing wind velocity $(P<0.05)$. The abundance of potentially beneficial bacteria showed differedamong the wind treatments $(P<0.05)$. The large fluctuations in the amount of bacteria provided a breeding opportunity forpotentially harmful bacteria $(P<0.05)$. In addition, there were significant decreases in the serum levels of IL-2 and IFN- $y(P<0.05)$ and a large increase in IL-4 level $(P<0.05)$, which indicated that the sheep underwent immune suppressionduring the trial. The significant increase in the activities of the antioxidant enzymes SOD, GSH-PX, and CAT $(P<0.05)$ indicated that the production of oxygen free radicals was increased.

Conclusions: The cold environment significantly reduced the growth of sheep and altered the composition of rumen microbiota, reducing the utilization of soluble fiber by the rumen flora. Furthermore, the sheep produced large amounts of enzymes to resist tissue damage and experienced immune suppression in the cold environment.

\section{Background}

Climate change can cause stress responses in animals[1], especially sharp decreases in temperature. In cold environments, the weight of livestock tends to be reduced. It has been found that cold exposure alters the composition of the intestinal microbiota by affecting food intake[2, 3], which could cause other changes in animal phenotype.

Studies in many monogastric animal models have investigated correlations between the intestinal microbiota and host phenotype[4]. In ruminants, the rumen is a large fermentation site containing microbes. Similar to the intestinal microbiota, the rumen microbiota could interact with the host[5]. Furthermore, the host could limit the abundance and community composition of rumen microbes to maintain homeostasis[6]. In turn, the rumen microbiota composition could affect inflammation and 
oxidation, which can be measured by inflammatory-related markers, such as interleukin-2 (IL-2), interleukin-4 (IL-4), interleukin-6 (IL-6), and interferon-y (IFN-ץ), and markers of oxidative stress, such as malondialdehyde (MDA) and various antioxidant enzymes, respectively. The rumen epithelium could produce hormones that influence phenotype, such as peptide-1, peptide YY, ghrelin, and leptin[7, 8]. Changes in the rumen microbiota could affect metabolites and the energy supply to tissues by producing volatile fatty acids (VFAs) by fermenting carbohydrates and inflammatory reaction[7, 9, 10]. Cold exposure could lead to changes in the intestinal microbiota in monogastric animals and affect host oxidation and inflammation[11]. Many studies have shown that cold exposure could alter the intestinal microbiota. However, the effects of a cold environment on the rumen microbiota are unclear.

High-altitude areas in the Northern Hemisphere experience low temperatures in winter, which reduce the productivity and feed-utilization efficiency of livestock and severely constrain the economic benefits of animal husbandry[12]. In northern China, the cold winter temperatures are always accompanied by strong northwest winds. Wind and low temperature aggravate the convection and heat dissipation of the body, imposing cold stress on livestock. In this experiment, we varied wind speed to investigate how cold exposure affects rumen microbiota and metabolism in sheep. We hypothesized that a cold environment affects the rumen microbiota and that changes to their metabolites affect host phenotype in ruminants.

\section{Material And Methods}

\section{Ethical approval}

All the animal procedures were carried out in accordance with the guidelines of the China Council on Animal Care and the Ministry of Agriculture of the People's Republic of China. The use of animals and all experimental protocols (protocol number 100403) were authorized by the Institutional Animal Care and Use Committee of Northwest A\&F University (Yangling, Shaanxi, China).

\section{Experimental animals and design}

In this experiment, the test site and the corresponding test materials were provided by Linze Experimental Station of Lanzhou University, China. Twelve healthy 6-month-old crossbred hybrid ewes (small-tailed Han sheep $\times$ Hu sheep; mean body weight (BW) $30.36 \pm$ SE 1.68 kg; not pregnant before the experiment) were randomly allocated into three groups: I, II and III (four sheep per group).

The experiment spanned 20 days, comprising 10 days for adaptation and 10 days for the trial period. The sheep were divided into a control group and treatment groups. Andthe experimental condition other than wind sheep were the same across the treatment groups (including feeding management, test time, time of use of metabolic cages, etc.). According to records of annual average wind speed in the Atlas of Natural Disaster Systems in China, the annual average wind speed in the study area is approximately $3 \sim 4 \mathrm{~m} / \mathrm{s}$; this range was used to set the wind-speed gradient. Increasing wind speed increases thermal convection, exacerbating the effect of cold stress on sheep. The sheep in the treatment groups were subjected to one of four wind-speed treatments: no wind (average wind speed or less than $0.5 \mathrm{~m} / \mathrm{s}$, low temperature (LT)), 
low wind (average wind speed of $3 \mathrm{~m} / \mathrm{s}, \mathrm{LW}$ ), medium wind (average wind speed of $4 \mathrm{~m} / \mathrm{s}$, moderate wind $(\mathrm{MW})$ ) and high wind (average wind speed of $5 \mathrm{~m} / \mathrm{s}$, high wind (HW)).To reduce the influence of diurnal temperature variation on the results, wind-speed treatment was performed from 8:00 p.m. to 8:00 a.m. of the second day, and the rest of the time was used as a rest and recovery period.During the test period, the outdoor night average temperature was $-17^{\circ} \mathrm{C}$ and the average temperature in the sheep house was approximately $5^{\circ} \mathrm{C}$.

The test was carried out in two stages. In the first stage, the test sheep of groups I, II and III were allocated as the control group $(C)$, no wind under low temperature $(L T)$, and low windunder low temperature $(L W)$, respectively. In the second stage of the test, the sheep of groups II and III were treated with MW and HW, respectively, under LT. Stage 1 spanned days 10 to 15 , and stage 2 spanned day 16 to 20 . There was no interval between the two phases, which spanned 5 days each (See Additional file 1: Fig.S1). This procedure ensured that the test sheep gradually adapted to cold stress, which increased from low to high, avoiding injury or death due to direct exposure to high-intensity cold stress. In addition, compared with a sudden change, a gradual change in temperature more closely resembles temperature change in the field.

The wind treatments were carried out by industrial electric fan (fl65-1, Watson, China; maximum wind speed, $6.5 \mathrm{~m} / \mathrm{s}$ ). In each treatment, four fans were used to create wind in different directions to ensure a uniform wind speed. During the test period, a windproof barrier was built at the test site to avoid interference from external natural wind. The external wind speed was monitored throughout the experiment. Testo 405-v1 anemometer (testto405-v1,tmall, Germany) was used to measure the wind speed at different points in the sheepfold and calculate the average wind speed.

\section{Feeding and management}

To avoid excessive differences in wind speed among different places in the large sheepfold, two open sheep pens $(2.5 \times 2.5 \mathrm{~m})$ were set up in the sheepfold before the experiment began and sterilized. The experimental sheep were fed twice daily (at 9:00 a.m. and 6:00 p.m.), and some residual feed remained after each feeding, indicating that the sheep had been adequately fed.

The sheep were fed complete formula pellet feed (582 Formula Feed, Yuansheng, 120 China). The feed composition and nutrient levels are shown in Table S1.Before the test period, due to the low ambient temperature, the water in the water tank provided for drinking completely froze for approximately 1 to $2 \mathrm{~h}$ each day. Therefore, the water tank was regularly monitored during the study and any ice removed to ensure sheep access to drinking water.

\section{Sampling}

In each treatment group, the sheep were fed in a metabolic cage from the third day to the fifth day of treatment. The sheep were weighed without feeding at 8:00 a.m. before entering the metabolic cage, and the average daily gain (ADG) was calculated. In the morning of the 4th, 5th, and 6th days, all feces and urine were collected and weighed, and the daily fecal and urine volumes were recorded. The collected 
feces and urine were then stored at $-20^{\circ} \mathrm{C}$ in a refrigerator. The collected fecal samples and feed samples were dried in an oven at $65^{\circ} \mathrm{C}$ for $24 \mathrm{~h}$. The initial moisture was determined, and the dry fecal content and dry matter intake (DWI) were calculated. The dried fecal samples and feed samples were crushed into powder for testing.DWI, average daily weight gain, fecal volume, urine volume, and apparent digestibility of dry matter were measured.

On the last three days of every treatment, blood samples from all sheep were collected by jugular venipuncture into a serum separator tube andimmediately centrifuged at $3,000 \mathrm{~g}$ for $20 \mathrm{~min}$. The serum was then stored at $-40^{\circ} \mathrm{Cfor}$ the analysis of biochemical indicators. The levels of antioxidant enzymes, including superoxide dismutase (SOD), malondialdehyde (MDA), catalase (CAT) and glutathione peroxidase (GSH-PX); total antioxidant capacity (T-AOC); and the levels of immune factors IL-2, IL-4, IL-6, and IFN- $y$ were measured.The kits used to measure the indexeswere purchased from Beijing Huaying Biotechnology Research Institute, Beijing, China.The timeline of the experiment was shown (See Additional file 1: Fig.S1). On the last day of each treatment, after fasting the sheep overnight, the rumen contents were carefully pumped out, separated and stored at $-20^{\circ} \mathrm{C}$ for analysis.

\section{Total DNA extraction from rumen fluid and quantification PCR}

Total genomic DNA was extracted using the stool DNA kit (OMEGA Bio-Tek, Norcross, GA, USA) according to the manufacturer's instructions. The concentration and quality of DNA were measured using a K5800 microspectrophotometer (KAIAO, Beijing, China). Quantitative PCR was executed in triplicate using SYBR Premix Ex Taq II (RNaseH Plus) assay kit (TaKaRa Bio Inc., Kusatsu, Shiga, Japan). The reaction system and procedure followed a previous study[13]. The primer sequences were selected based on past research[14].

\section{S r RNA gene sequencing, data processing and functional prediction}

The V3-V4 region of the total microbial 16S r RNA gene was amplified using primers 338F(5'ACTCCTACGGGAGGCAGCAG-3') and 806R (5'-GGACTACHVGGGTWTCTAAT-3') that were tailed with specific sequences and amplified genes. Amplification was performed with the following cycling conditions: $95^{\circ} \mathrm{C}$ for $3 \mathrm{~min}$, followed by 27 cycles of $95^{\circ} \mathrm{C}$ for $30 \mathrm{~s}, 55^{\circ} \mathrm{C}$ for $30 \mathrm{~s}$, and $72^{\circ} \mathrm{Cfor} 45 \mathrm{~s}$, and a final extension at $72^{\circ} \mathrm{C}$ for $10 \mathrm{~min}[14]$. The products were separated on $2 \%$ agarose gel, and nucleotides were isolated via bead purification using the AxyPrep DNA Gel Extraction Kit (Axygen Biosciences, Union City, USA). Each product was assembled in equimolar amounts and sequenced on the Illumina MiSeq platform (Illumina, San Diego, USA).

The sequence data from 16S rRNAMiSeq sequencing were analyzed, quality-filtered using Trimmomatic, and merged by FLASH $[15,16]$. The RDP classifier can quickly and accurately classify sequences into high-order taxonomy, which can provide a range of classification structures from the domain to genus level and accurately evaluate each stage[15]. Reads of $97 \%$ similarity were clustered into operational taxonomic units (OTUs) with $\leq 1 \%$ incorrect bases using UPARSE[17]. Chimeric $16 \mathrm{~S}$ rRNA sequences were removed after CS detection[18]. QIIME can be used to analyze a microbial community and graphically 
display the results[19]. The functional prediction of rumen bacteria was performed based on previous work[14].

\section{Volatile fatty acid (VFA) analysis}

The rumen fluid was thawed on the ice and centrifuged to obtain the supernatant, which was stored at $4^{\circ} \mathrm{C}$. For SCFA analysis, a solution was prepared by mixing the supernatant and the crotonic acid at the ratio of 10:1 and then filtered and analyzed using gas chromatography (Agilent Technologies 7820A GC system, Santa Clara, USA) according to previous studies[20].

\section{Statistical analysis}

For each 16S rRNA sample, the abundances of Kyoto Encyclopedia of Genes and Genomes (KEGG) pathways were estimated by calculating the means and standard errors of the mean (SEM) using oneway ANOVA with SPSS version 17.0 (SPSS Inc.; Chicago, IL, USA). For the bacterial community, we performed alpha and beta diversity analyses; the alpha diversity indexes Simpson, Shannon, and Chao were calculated, and beta diversity was explored by PLS-DA graphs. Rarefaction curves were produced in $\mathrm{R}$ (version 3.5.1).

The data, including BW, antioxidant indexes, immune indexes, and SCFA concentrations, were analyzed using $\mathrm{R}$ (3.5.0). $\mathrm{R}$ was used to analyze the associationsbetweenmicrobial community composition and these factors[21]. Covariance analysis was used to determine the effect of cold stress (sheep number was included as a covariate). $\mathrm{R}$ was used to construct graphs. In addition, we performed correlation network analysis. All data are presented as the mean $\pm \mathrm{SE}$, and values of $P<0.05$ were considered statistically significant.

\section{Results}

\section{Growth performance and nutrient digestibility}

We measured and calculated some of the metrics to estimate the performance of the production quota. We found that the ADG of sheep significantly decreased $(P<0.05)$ with increasing intensity of cold stress, and after initiating wind treatment, sheep weight began to decrease (Fig. 1a).

To study whether changes in energy intake or energyoutput were responsible for the change in BW, sheep were fed in the metabolic cage. We found that Dry matter intake (DMI) varied among the treatment groups. DMI was low in the $\mathrm{C}$ group and lowest in the LT group. DMI then increased with increasing wind velocity, being similar between the LW and MW groups and highest in the HW group $(P<0.05)$ (Fig. 1b). The apparent digestibility of dry matter was much lower in LT and LW than $C(P<0.05)$, whereas that in MW and HW was similar to that in C(Fig. 1C). As cold stimulation increased, the amount of crude fiber (CF) in feces increased significantly (Fig. 1d), possibly due to the degradation of primarily carbohydrate rather than cellulose by the rumen microbiota in the MW and HW groups. The levels of metabolic energy and digestibility energy were significantly lower in the LT and LW groups than the other groups (See 
Additional file 1: Fig.S2). These data indicated that the cold environment led to weight loss in the sheep and reduced digestion of the fiber in their feed.

\section{Rumen microbiota changes with environmental changes}

We used 16S rRNA gene sequence technology to analyze the abundance of rumen microbiota. The coverage index indicated that cold temperatures influenced microbial diversity (See Additional file 2: Table S1, S2). Through partial least squares discriminant analysis (PLS-DA), we found that microbiota community structures differed among the treatments(Fig. 2a).

We investigated the a diversity of the microbiota. Eighteen taxa were identified at the phylum level. Among the phyla, Bacteroidetes $(58.7962 \pm 2.0898 \%)$ had the highest diversity, followed by Firmicutes (34.2305 $\pm 1.6897 \%)$, Proteobacteria(2.7963 $\pm 0.8040 \%)$, and Fibrobacteres $(1.0466 \pm 0.2894 \%)$. The remaining taxa had values less than $1 \%$. The diversity of Verrucomicrobia $(0.3078 \pm 0.0486 \%)$ was significantly higher in the HW group than in the $C$ and LT groups $(P=0.043)$.

At the genus level, 216 taxa were identified. Univariate ANOVA of the bacterial abundances revealed several significant differences in the rumen microbes among treatments(Fig. 2). The relative abundance of Prevotellaceae_UCG-003(Bacteroidetes, $P=0.045$ ) and Solobacterium(Firmicutes, $P=0.017$ ) were decreased in the LT, LW, MW and HW groups compared with the $\mathrm{C}$ group. Furthermore, the abundance of Brachybacterium(Actinobacteria, $P=0.040)$, Devosia(Proteobacteria, $P=0.008$ ), Sphingomonas(Proteobacteria, $P=0.000$ ) and unclassified_f_Enterobacteriaceae(Proteobacteria, $P=$ $0.050)$ were higher in the LT group than in the other groups. The abundance of Rhizobium(Proteobacteria, $P=0.007$ )was higher in the LT and LW groups than the other groups. In contrast, the abundance of Sphaerochaeta(Spirochaetae, $P=0.044$ ) was decreased in the LT and LW groups compared with the other groups. Furthermore, the abundance of Pseudobutyrivibrio(Firmicutes, $P$ $=0.039)$ was higher in the MW group than in the other groups. The abundance of Ruminiclostridium_1(Firmicutes, $P=0.020$ ), Ruminococcaceae_UCG-005(Firmicutes, $P=0.044$ ), norank_c_WCHB1-41(Verrucomicrobia, $P=0.043$ ) was increased in the HW group relative to the other groups. Interestingly, the abundance of Lachnospiraceae_XPB1014(Firmicutes, $P=0.012$ )exhibited highest levels in the LT group and the lowest levels in the LW group (Fig. 2).

We used qPCR to verify the changes in some bacterial groups (See Additional file 2: Table S3). The diversity of the dominant bacteriadid not differ significantly (See Additional file 2: Table S4).

\section{Bacterial function prediction and molecular pathways in the rumen}

We predicted the functions of the rumen bacteria and the associated molecular pathways in sheep to assess the impact of wind treatment. At KEGG level 1, there were 7 major categories, including Metabolism (49.76 $\pm 0.22 \%)$, Genetic Information Processing (21.32 $\pm 0.06 \%)$, Unclassified (13.95 $\pm 0.04 \%)$, Environmental Information Processing (10.58 $\pm 0.23 \%)$, Cellular Processes $(2.68 \pm 0.08 \%)$, Organism System $(0.77 \pm 0.01 \%)$, and Human Diseases $(0.75 \pm 0.01 \%)$. The gene abundance of Unclassified was 
significantly lower in the HW group than the other groups $(P<0.05)$; no other significant differences were observed (See Additional file 2: Table S5).

At KEGG level 2, a large percentage of 41 gene families were found to have correlations with Amino Acid Metabolism (10.57 $\pm 0.56 \%)$, Carbohydrate Metabolism (10.12 $\pm 0.05 \%)$, Replication and Repair (9.88 $\pm 0.04 \%)$, Membrane Transport $(9.13 \pm 0.21 \%)$, Translation $(6.00 \pm 0.02 \%)$, andEnergy Metabolism $(6.03 \pm 0.03 \%)$ (See Additional file 2: Table S6). The gene abundance of Nervous System was significantly lower in the MW group than in the other groups $(P<0.05)$. However, the gene abundance of Excretory System was lower in the LT, LW, and MW groups than in the $C$ and HW groups, which had similar abundance $(P=0.06)$. At KEGG level $3,328 \mathrm{KEGG}$ orthology $(\mathrm{KO})$ pathways were identified. The top 44 pathways with high expression are shown (See Additional file 2: Table S7). The gene abundance did not differ significantly among groups. These findings showed that LT and wind speed may affect the nervous system in the rumen.

\section{Changes in the concentration of VFAs and cellulase activity in the rumen}

Studies have shown that VFAs could provide energy to the host and participate in the host metabolism[4]. After discovering the changes in the rumen microbiota, we explored the levels of VFAs. We found that the concentration of total VFAs was significantly reduced in the MW and LW groups relative to the other groups $(P<0.05)$ (Fig. 3a). Accordingly, the concentrations of acetic acid and propionic acid were decreased significantly in the MW and HW groups $(P<0.05)$. However, the ratio of acetic acid to propionic acid did not markedly differ among the groups (See Additional file 2:Table S8). In addition, butyrate level was significantly reduced in the HW group relative to the other groups $(P<0.05)$, whereas isobutyricacid and isovalerate levels were significantly increased in the MW and HW groups compared with the other groups $(P<0.05)$.Cellulase activity in the rumen contents decreased with increasing wind speed, being significantly lower in the HW group than in the other groups $(P<0.05)$ (Fig. 3b). These findings suggested that in the cold environment, the rumen microbes reduced their digestion of CF.

\section{Changes in inflammatory factors and antioxidant enzymes}

As we expected, the contents of proinflammatory factors, such as IL-2, IL-6, and IFN- $y$, in plasma were reduced in the wind-treatment groups relative to the $C$ group $(P<0.05)$. In contrast, the contents of antiinflammatory factors, such as IL-4, were increased in the wind-treatment groups relative to the $\mathrm{C}$ group $(P<0.05)$ (Fig. 4).

We found that MDA content was significantly decreased in the wind-treatment groups compared with the C group $(P<0.05)$ (Fig. 5a). In contrast, the serum concentrations of SOD, CAT and GSH-PX showed similar trends as T-AOC (Fig. 5), being increased in the wind-treatment groups relative to the $C$ group $(P<0.05)$. The ratio of T-AOC to MDA reflects the relationship between the body's antioxidant capacity and oxidative damage. Low-temperature treatment significantly increased the ratio of T-AOC to MDA in serum $(P<0.05)$, and this ratio increased significantly with increasing WV $(P<0.05)$. However,the ratio of T-AOC to MDAwas significantly lower in theLW group than in all of the other groupsexcept the $C$ group, for which 
no significant difference was observed. These data showed that cold stimulation led the sheep to enter an immunosuppressive and antioxidant state.

\section{Associations of rumen microbiota with host phenotype}

We used correlation analysis to research the associations between microbiota and host phenotype (Fig. 6 , Additional file 3: Table S9). ADG was negatively correlated with Ruminiclostridium_1 $(\mathrm{r}=-0.525, P<0.05)$, Ruminococcaceae_UCG-005 $(r=-0.480, P<0.05)$, Sphaerochaeta $(r=-0.479, P<0.05)$, and norank_c_WCHB1-41 $(r=-0.519, P<0.05)$ and the levels ofisobutyric acid $(r=-0.500, P<0.01)$ and isovalerate $(r=-0.553, P<0.05)$. Positive relationships were detected between ADG and Solobacterium $(r=$ $0.583, P<0.01)$ and the levels ofacetic acid $(r=0.583, P<0.01)$, propionic acid $(r=0.523, P<0.05)$, butyrate $(r=0.638, P<0.01)$, valeric acid $(r=0.521, P<0.05)$, and total VFA $(r=0.499, P<0.05)$.

In addition, we found that some microbiota were associated with the levels of certain inflammatory factors. For example, the levels of the proinflammatory factors IL-2 and IFN- $\gamma$ were positively associated with Prevotellaceae_UCG-003 $(r=0.618, P<0.01 ; r=0.708, P<0.01$.respectively) andSolobacterium $(r=$ $0.541, P<0.05 ; \mathrm{r}=0.675, P<0.01$.respectively). Furthermore, a significant negative association was detected between IFN-y level and norank_C__WCHB1-41 ( $\mathrm{r}=-0.494, P<0.05)$, and a positive relationship was found between IL-4 leveland Lachnospiraceae_XPB1014 ( $r=553, P<0.05)$.

Furthermore, correlations between microbiota and oxidative stress markers were identified.TPrevotellacee_UCG-003had a significant positive correlation with MDA level $(r=0.534, P]$ $0.05)$ and negative correlations with T-AOC $(r=-0.451, P<0.05)$, SOD $(r=-0.646, P<0.01)$, CAT $(r=-0.664$, $P<0.01)$, and GSH-PX levels $(r=-0.532, P<0.05)$. TSphingomonashad significant positive correlations with T-AOC $(r=0.602, P<0.01)$, CAT $(r=0.608, P<0.01)$, and GSH-PX levels $(r=0.506, P<0.05)$

\section{Discussion}

Winter in northwest China is not only cold but also subject to strong winds. We used the local temperature in this study and strictly controlled the wind speed. In the present study, we explored the whole-body and rumen responses of sheep to both cold and wind speed. When the sheep were exposed to cold temperature, DMIdecreased sharply. In contrast, Bo reported that voles exposed to cold temperature $\left(4^{\circ} \mathrm{C}\right)$ increased their food intake[22] to maintain a constant body temperature. However, in the present study, the range of temperature was very large. The sharp decrease in BMI with cold exposure observed in the present study corresponds to the first stage in the stress response, i.e., a panic reaction or mobilization phase, as proposed by Canadian pathologist Hans Selye. This reaction led to a decline in animal feed intake. In addition,the metabolic analyses revealed that the apparent digestibility of DM, DE, and MEdecreased sharplyupon cold exposure. Young et al concluded that each $10^{\circ} \mathrm{Cdecrease}$ in an environment below $20^{\circ} \mathrm{C}$ would cause 1.8 percentage points of DM digestibility change[23], reducing the feed-utilization efficiency of sheep. This conclusion is consistent with our results. Subsequently, wind treatment was applied to the sheep in the cold environment. Under wind exposure, the sheep increased the apparent digestibility of DM, DE, and ME. They then entered the second phase of the stress response: 
the adaptation phase. They generated more heat from feeding and the body to maintain a constant body temperature. As has been described in previous studies, the ADG of sheep decreased significantly under wind treatment and became negative[22].

The rumen microbiota change when animals are exposed to cold conditions[2]. For example, when the sheep were subjected to cold treatment, the abundance of Lachnospiraceae_XPB1014, which is highly enriched in the gut of nonalcoholic fatty liver patients[24], increased, and the abundance of Prevotellaceae_UCG003 decreased. These two bacterial taxa represent more than $1 \%$ of the bacterial community in the rumen anduse dietarysoluble fiber as substrate to produce short chain fatty acids[25].Furthermore, several nitrogen-fixing microbial groups, such asDevosia and Rhizobium[26], were enriched in the wind-exposed sheep, which might increase the amount of ammonia and urea produced via the rumen nitrogen cycle. As a result, the abundance of Brachybacterium increased, which uses urea to breed and degrade harmful substancessuch asphenol[27]. In addition, the abundance of Sphingomonas, which is involved in redox reactions and has a reducing effect[28], was increased under cold temperature.Large fluctuations in the amount of bacteria in the rumen provide a breeding opportunity for pathogens such as unclasssified_f_Enterobacteriaceae[29]. Treatments with different wind velocities were applied to sheep. As WV increased, the abundances of Lachnospiraceae_XPB1014, Ruminiclostridium_1, Ruminococcaceae_UCG005 and norank_f_WCHB1-41 increased.

Lachnospiracea__XPB1014 digests soluble fiber,theother two groups had abundances between $0.1 \%$ and $1 \%$ and degrade cellulose[30-32]. Furthermore, the abundance of Pseudomonasincreased, which might reduce oxidative stress[33]. We concluded that after cold stimulation, rumen bacteria that digest soluble fiber fluctuated in abundance, while the abundances of beneficial bacteria decreased and those of harmful bacteria increased.

PICRUSt1 functional prediction revealed that the rumen microbiota were regulated by the nervous system under cold temperature. Several studies have found that the gut microbiota in mice are regulated by neurons, such as VIP neurons[34]. We suspect that rumen microbes can similarly be regulated by neuronal factors. Changes in the rumen microorganisms caused changes in VFAs. Consistent with our findings, previous studies have found that cold conditions reduce the contents of total VFA, acetate, butyrate, and valerate[12]. These reductions occur due to the lower efficiency of rumen microorganisms in fermenting soluble fibers in cold conditions[35].

Some studies have shown that animals exposed to cold temperature can enter a severe inhibitory stateof the immune response[36, 37].This observation is consistent with our findings. IL-2, which could represent the level of cellular immunity[38], and IFN- $\gamma$, which is mainly involved in cellular immune-related immune responses, were decreased in the sheep exposed to cold temperature. Furthermore, the level of IL-4, which could represent the activation level of TH2 cells, was increased in these sheep. Following these changes,the content of TH2 likely increased sharply and that of TH1 likely decreased[39], which destroyed the normal dynamic equilibrium state of the two types of cells, causing the body to enter an immunosuppressive state. In addition, the serum content of the proinflammatory factor IL- 6 was decreased in the cold-treated sheep in this study. Inconsistent with our results, Guo et al found that IL-6 
levels increased under cold conditions[40].The difference may be due to study differences in the levels of cold stimulation in the experimental design and in the genetic backgrounds of the animals. The immune response is very complex, and proinflammatory factors are affected by many factors[34], which deserve further study.

When the sheep were exposed to cold temperature,they metabolized large amounts of nutrients to increase their bodies' heat production.During this process, the body could produce oxygen free radicals, resulting in oxidative damage[41]. However, in the present study,MDA content,which reflects the extent of cell damage[42], was decreased in the cold-exposed sheep. We speculate that the high levels of antioxidant enzymes, such as SOD[43], CAT[44], and GSH-PX[45], in plasma under cold temperature led to the degradation of the oxygen free radicals produced by sheep metabolism[46]. Studies have shown that low levels of cold stress enhance antioxidant capacity and reduce the body's exposure to the effects of oxidative stress[47]. Furthermore, studies have shown that elevated levels of antioxidant enzymes in sheep are indicative of oxidative stress, with high enzyme levels needed to avoid damage fromoxygen free radicals[45]. These observations are consistent with our findings.

Accumulating studies have shown that microbes have significant associations with and can alter host phenotypes[48, 49]. VFA content was strongly correlated with ADG in this study. VFAs, especially butyrate[35], can provide large amounts of energy to fuel metabolism. However, the mechanismsunderlying the associationsbetween specific microorganisms and ADG need further study. Some studies have shown that the abundances of Prevotellaceae_UCG003[25] and Lachnospiraceae_ $X P B 1014$,which produce metabolites such as VFAs[50],influence the immune response. In addition, the abundance of Sphingomonas, which is involved in reduction reactions, has been shown to be increased in the rumen of animals subjected to cold stress[51]. The increase in Sphingomonas abundance resulted in a decrease in MDA content and a positive correlation with blood hormone content, consistent with our results. However, the associations between Prevotellaceae_UCGOO3 and blood hormones indicative of oxidative damage deserve further study.

\section{Conclusion}

In the present study, when the sheep were exposed to the cold environment, animal growth and feed efficiency decreased significantly, and the fermentation of soluble fibers by rumen microorganisms decreased significantly. In addition, sheep increased the levels of antioxidant enzymes to resist damage; however, the sheep were in astate of immunosuppression.

\section{Abbreviations}

ADG: average daily gain; SOD: superoxide dismutase; GSH-PX: glutathione peroxidase; CAT: catalase; VFA: volatile fatty acid; BW: body weight; MDA: malondialdehyde; T-AOC: total antioxidant capacity; KEGG: Kyoto Encyclopedia of Genes and Genomes; SEM: standard error of the mean; DMI: dry matter intake; WV: wind velocity; DM: dry matter; CF: crude fiber; PLS-DA: partial least squares discriminant 
analysis; PICRUSt2: Phylogenetic Investigation of Communities by Reconstruction of Unobserved States;qPCR: quantitative real-time polymerase chain reaction;SPSS: Statistical Package for the Social Sciences; ANOVA: one-way analysis of variance

\section{Declarations}

\section{Ethics approval and consent to participate}

All procedures in the present study involving animals were approved by the Animal Care and Use Committee of Lanzhou University (Lanzhou, China) and Northwest A\&F University (Yanglin China).

\section{Consent for publication}

Not applicable

\section{Availability of data and materials}

Raw Illumina sequencing data have been deposited in Sequence Read Archive (SRA). BioProject's metadata are available at the following link:

http://www.ncbi.nlm.nih.gov/bioproject/633534

The other datasets analyzed in the current study are available from the corresponding author upon reasonable request.

\section{Competing interests}

The authors declare no competing financial interests.

\section{Funding}

The study was financially supported by the National Key Research and Development Program of China (2016YFD0500508) and the China Agriculture Research System (CARS-39-12).

\section{Authors' contributions}

YY and $C Y$ conceived and designed the experiments. ZG,ZS, TG and LY managed the sheep. ZG,CH,TG and $\mathrm{LY}$ collected samples. $\mathrm{GH}$ and $\mathrm{DN}$ performed the bacterial analysis. $\mathrm{GH}$ and $\mathrm{ZG}$ analyzed the other data.ZG and GH performed the statistical analyses, and GH and ZG wrote the manuscript. All authors read and approved the final version of the manuscript.

\section{Acknowledgments}

The authors are grateful to the staff of Linze station of Lanzhou University for their assistance in conducting the experiments. We also thank Yaoyue Wang, Xiaoyu Huang, Yu Zhang, and Xiaokun Peng 
for their diligent contributions to the animal experiments.

\section{Author details}

College of Animal Science and Technology, Northwest A\&F University, Yangling712100, People's Republic of China

\section{References}

1. Fan C, Su D, Tian H, Li X, Li Y, Ran L et al. Liver metabolic perturbations of heat-stressed lactating dairy cows. Asian-Australas J Anim Sci. 2018; 31:1244-1251.

2. Chevalier C, Stojanovic O, Colin DJ, Suarez-Zamorano N, Tarallo V, Veyrat-Durebex C et al. Gut Microbiota Orchestrates Energy Homeostasis during Cold. Cell. 2015; 163:1360-1374.

3. Virtue AT, McCright SJ, Wright JM, Jimenez MT, Mowel WK, Kotzin JJ et al. The gut microbiota regulates white adipose tissue inflammation and obesity via a family of microRNAs. Sci Transl Med. 2019; 11.

4. Oliphant K, Allen-Vercoe E. Macronutrient metabolism by the human gut microbiome: major fermentation by-products and their impact on host health. Microbiome. 2019; 7:91.

5. Tipton L, Darcy JL, Hynson NA. A Developing Symbiosis: Enabling Cross-Talk Between Ecologists and Microbiome Scientists. Front Microbiol. 2019; 10.

6. Litvak Y, Baumler AJ. Microbiota-Nourishing Immunity: A Guide to Understanding Our Microbial Self. Immunity. 2019; 51:214-224.

7. Clarke G, Stilling RM, Kennedy PJ, Stanton C, Cryan JF, Dinan TG. Minireview: Gut microbiota: the neglected endocrine organ. Mol Endocrinol. 2014; 28:1221-1238.

8. Possemiers S, Bolca S, Verstraete W, Heyerick A. The intestinal microbiome: a separate organ inside the body with the metabolic potential to influence the bioactivity of botanicals. Fitoterapia. 2011; 82:53-66.

9. Belkaid Y, Hand TW. Role of the microbiota in immunity and inflammation. Cell. 2014; 157:121-141.

10. Rafiki A, Boulland JL, Halestrap AP, Ottersen OP, Bergersen L. Highly differential expression of the monocarboxylate transporters MCT2 and MCT4 in the developing rat brain. Neuroscience. 2003; 122:677-688.

11. Fu J, Liu CP, Zhang ZW, Xing MW, Xu SW. Influence of inflammatory pathway markers on oxidative stress induced by cold stress in intestine of quails. Res Vet Sci. 2013; 95:495-501.

12. Liang X, Jin J, Bi X, Kamruzzaman M, Kudo T, Sano H. Effects of Chinese herbal medicine and cold exposure on plasma glucose, leucine and energy metabolism in sheep. J Anim Physiol Anim Nutr (Berl). 2018; 102:e534-e541.

13. Wang Y, Cao P, Wang L, Zhao Z, Chen Y, Yang Y. Bacterial community diversity associated with different levels of dietary nutrition in the rumen of sheep. Appl Microbiol Biotechnol. 2017; 101:37173728 . 
14. Zhong S, Ding Y, Wang Y, Zhou G, Guo H, Chen Y et al. Temperature and humidity index (THI)-induced rumen bacterial community changes in goats. Appl Microbiol Biotechnol. 2019; 103:3193-3203.

15. Magoc T, Salzberg SL. FLASH: fast length adjustment of short reads to improve genome assemblies. Bioinformatics. 2011; 27:2957-2963.

16. Zhao MM, Du SS, Li QH, Chen T, Qiu H, Wu Q et al. High throughput 16SrRNA gene sequencing reveals the correlation between Propionibacterium acnes and sarcoidosis. Resp Res. 2017; 18.

17. Edgar RC. UPARSE: highly accurate OTU sequences from microbial amplicon reads. Nat Methods. 2013; 10:996.

18. Haas BJ, Gevers D, Earl AM, Feldgarden M, Ward DV, Giannoukos G et al. Chimeric 16S rRNA sequence formation and detection in Sanger and 454-pyrosequenced PCR amplicons. Genome Res. $2011 ; 21: 494-504$.

19. Caporaso JG, Kuczynski J, Stombaugh J, Bittinger K, Bushman FD, Costello EK et al. QIIME allows analysis of high-throughput community sequencing data. Nat Methods. 2010; 7:335-336.

20. Li F, Li Z, Li S, Ferguson JD, Cao Y, Yao J et al. Effect of dietary physically effective fiber on ruminal fermentation and the fatty acid profile of milk in dairy goats. J Dairy Sci. 2014; 97:2281-2290.

21. Kaliannan K, Robertson RC, Murphy K, Stanton C, Kang C, Wang B et al. Estrogen-mediated gut microbiome alterations influence sexual dimorphism in metabolic syndrome in mice. Microbiome. $2018 ; 6: 205$.

22. Bo TB, Zhang XY, Wen J, Deng K, Qin XW, Wang DH. The microbiota-gut-brain interaction in regulating host metabolic adaptation to cold in male Brandt's voles (Lasiopodomys brandtii). ISME J. 2019; 13:3037-3053.

23. Young BA. Ruminant cold stress: effect on production. J Anim Sci. 1983; 57:1601-1607.

24. Shen F, Zheng RD, Sun XQ, Ding WJ, Wang XY, Fan JG. Gut microbiota dysbiosis in patients with nonalcoholic fatty liver disease. Hepatobiliary Pancreat Dis Int. 2017; 16:375-381.

25. Borgo F, Garbossa S, Riva A, Severgnini M, Luigiano C, Benetti A et al. Body Mass Index and Sex Affect Diverse Microbial Niches within the Gut. Front Microbiol. 2018; 9.

26. Willems A. The taxonomy of rhizobia: an overview. Plant Soil. 2006; 287:3-14.

27. Chou JH, Lin KY, Lin MC, Sheu SY, Wei YH, Arun AB et al. Brachybacterium phenoliresistens sp. nov., isolated from oil-contaminated coastal sand. Int J Syst Evol Microbiol. 2007; 57:2674-2679.

28. Kudlich M, Keck A, Klein J, Stolz A. Localization of the enzyme system involved in anaerobic reduction of azo dyes by Sphingomonas sp. strain BN6 and effect of artificial redox mediators on the rate of azo dye reduction. Appl Environ Microb. 1997; 63:3691-3694.

29. Dallenne C, Da Costa A, Decre D, Favier C, Arlet G. Development of a set of multiplex PCR assays for the detection of genes encoding important beta-lactamases in Enterobacteriaceae. J Antimicrob Chemoth. 2010; 65:490-495.

30. Yoav S, Barak Y, Shamshoum M, Borovok I, Lamed R, Dassa B et al. How does cellulosome composition influence deconstruction of lignocellulosic substrates in Clostridium 
(Ruminiclostridium) thermocellum DSM 1313. Biotechnol Biofuels. 2017; 10.

31. Zhao L, Zhang Q, Ma WN, Tian F, Shen HY, Zhou MM. A combination of quercetin and resveratrol reduces obesity in high-fat diet-fed rats by modulation of gut microbiota. Food Funct. 2017; 8:46444656.

32. Ji Y, Liu PF, Conrad R. Response of fermenting bacterial and methanogenic archaeal communities in paddy soil to progressing rice straw degradation. Soil Biol Biochem. 2018; 124:70-80.

33. Kang YS, Kim YJ, Jeon CO, Park W. Characterization of naphthalene-degrading Pseudomonas species isolated from pollutant-contaminated sites: Oxidative stress during their growth on naphthalene. J Microbiol Biotechn. 2006; 16:1819-1825.

34. Talbot J, Hahn P, Kroehling L, Nguyen H, Li D, Littman DR. Feeding-dependent VIP neuron-ILC3 circuit regulates the intestinal barrier. Nature. 2020; 579:575-580.

35. Koh A, De Vadder F, Kovatcheva-Datchary P, Backhed F. From Dietary Fiber to Host Physiology: ShortChain Fatty Acids as Key Bacterial Metabolites. Cell. 2016; 165:1332-1345.

36. Lavoy ECP, McFarlin BK, Simpson RJ. Immune Responses to Exercising in a Cold Environment. Wild Environ Med. 2011; 22:343-351.

37. Kozyreva TV, Eliseeva LS. Immune response in cold exposures of different types. J Therm Biol. 2000; 25:401-404.

38. Kandasamy M, Selvakumari Jayasurya A, Moochhala S, Huat Bay B, Kun Lee Y, Mahendran R. Lactobacillus rhamnosus GG secreting an antigen and Interleukin-2 translocates across the gastrointestinal tract and induces an antigen specific immune response. Microbiol Immunol. 2011; 55:704-714.

39. Becker Y. Respiratory syncytial virus (RSV) evades the human adaptive immune system by skewing the Th1/Th2 cytokine balance toward increased levels of Th2 cytokines and IgE, markers of allergya review. Virus Genes. 2006; 33:235-252.

40. Guo JR, Li SZ, Fang HG, Zhang X, Wang JF, Guo S et al. Different Duration of Cold Stress Enhances Pro-Inflammatory Cytokines Profile and Alterations of Th1 and Th2 Type Cytokines Secretion in Serum of Wistar Rats. J Anim Vet Adv. 2012; 11:1538-1545.

41. Neish AS. Redox signaling mediated by the gut microbiota. Free Radical Res. 2013; 47:950-957.

42. Kwiecien S, Konturek PC, Sliwowski Z, Mitis-Musiol M, Pawlik MW, Brzozowski B et al. Interaction Between Selective Cyclooxygenase Inhibitors And Capsaicin-Sensitive Afferent Sensory Nerves In Pathogenesis Of Stress-Induced Gastric Lesions. Role Of Oxidative Stress. J Physiol Pharmacol. 2012; 63:143-151.

43. Fukai T, Siegfried MR, Ushio-Fukai M, Cheng Y, Kojda G, Harrison DG. Regulation of the vascular extracellular superoxide dismutase by nitric oxide and exercise training. J Clin Invest. 2000; 105:1631-1639.

44. Switala J, Loewen PC. Diversity of properties among catalases. Arch Biochem Biophys. 2002; 401:145-154. 
45. Hong JH, Kim KJ, Suzuki K, Lee IS. Effect of cold acclimation on antioxidant status in cold acclimated skaters. J Physiol Anthropol. 2008; 27:255-262.

46. Cong P, Liu Y, Liu N, Zhang Y, Tong C, Shi L et al. Cold exposure induced oxidative stress and apoptosis in the myocardium by inhibiting the Nrf2-Keap1 signaling pathway. BMC Cardiovasc Disord. 2018; 18:36.

47. Li JH, Huang FF, Li X, Su YY, Li HT, Bao J. Effects of intermittent cold stimulation on antioxidant capacity and mRNA expression in broilers. Livest Sci. 2017; 204:110-114.

48. Plovier H, Everard A, Druart C, Depommier C, Van Hul M, Geurts L et al. A purified membrane protein from Akkermansia muciniphila or the pasteurized bacterium improves metabolism in obese and diabetic mice. Nat Med. 2017; 23:107-113.

49. Hu J, Ma LB, Nie YF, Chen JW, Zheng WY, Wang XK et al. A Microbiota-Derived Bacteriocin Targets the Host to Confer Diarrhea Resistance in Early-Weaned Piglets. Cell Host Microbe. 2018; 24:817.

50. Spencer SP, Fragiadakis GK, Sonnenburg JL. Pursuing Human-Relevant Gut Microbiota-Immune Interactions. Immunity. 2019; 51:225-239.

51. Busse HJ, Kampfer P, Denner EBM. Chemotaxonomic characterisation of Sphingomonas. J Ind Microbiol Biot. 1999; 23:242-251.

\section{Figures}




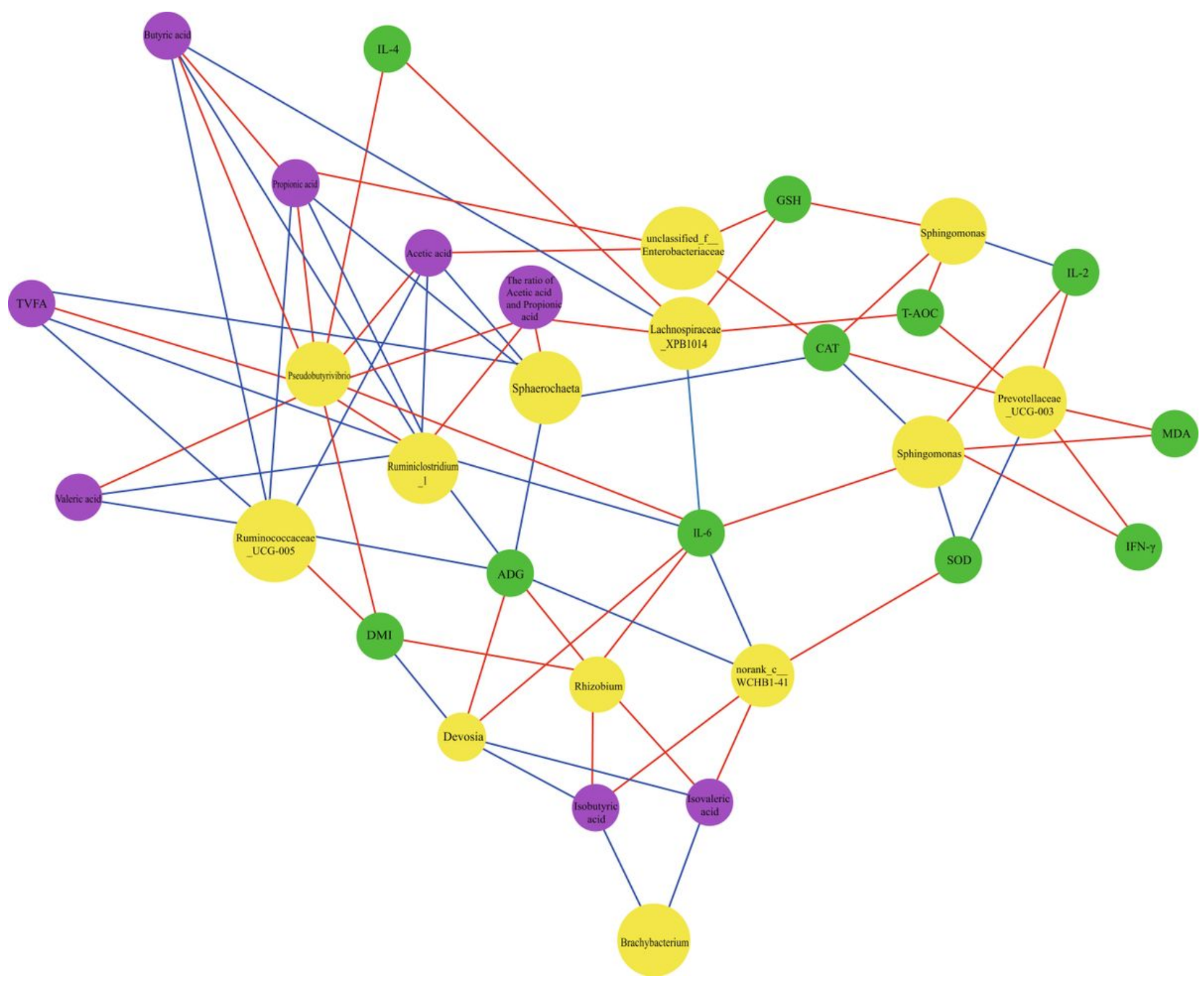

Figure 1

Correlation analysis of rumen microbiota and host phenotype. 
A

MDA

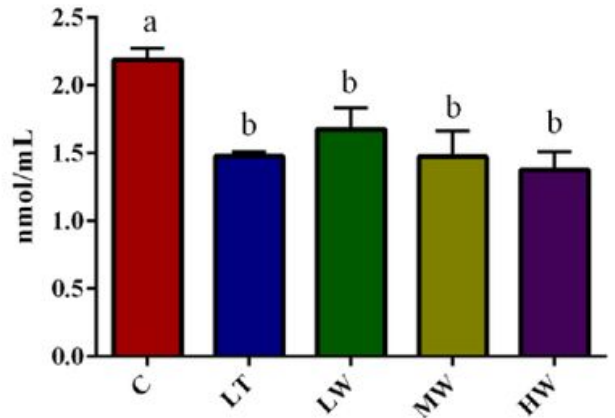

D

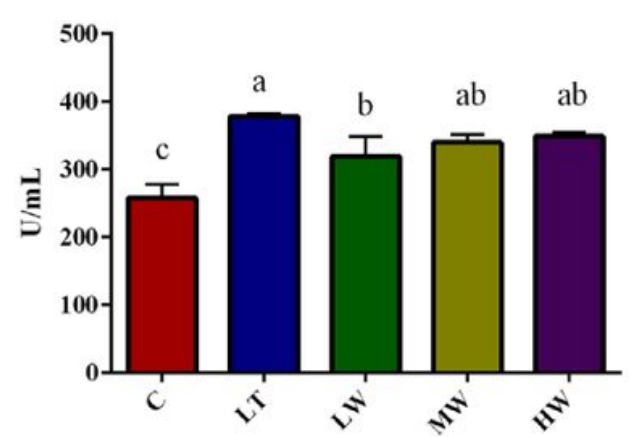

B

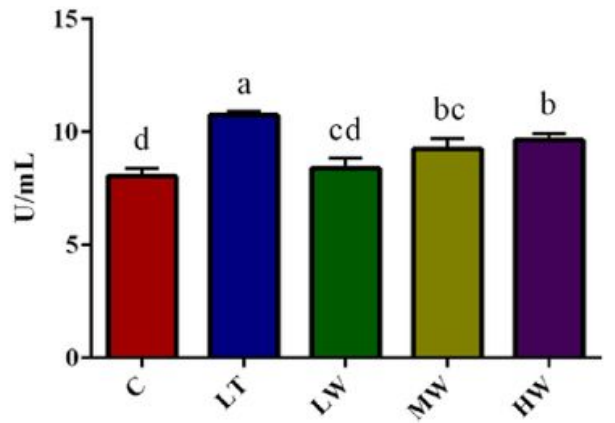

E

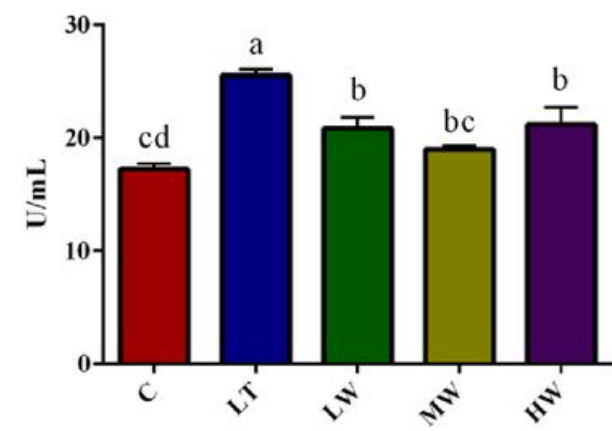

C

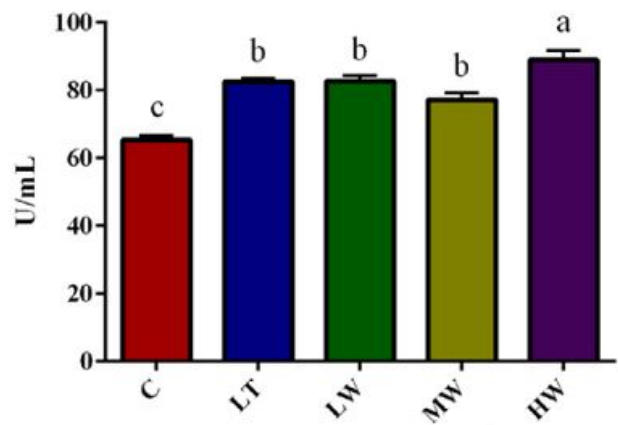

F

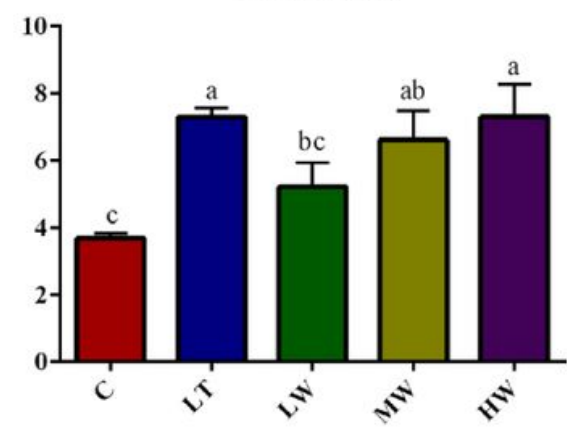

Figure 2

Effect of wind velocity on antioxidant indexes in sheep under low temperature. (A) Effect of wind velocity on MDA content in serum in sheep under low temperature. (B)Effect of wind velocity on T-AOC level in serum in sheep under low temperature. (C) SOD. (D) GSH-PX. (E) CAT. (F) T-AOC/MDA. 


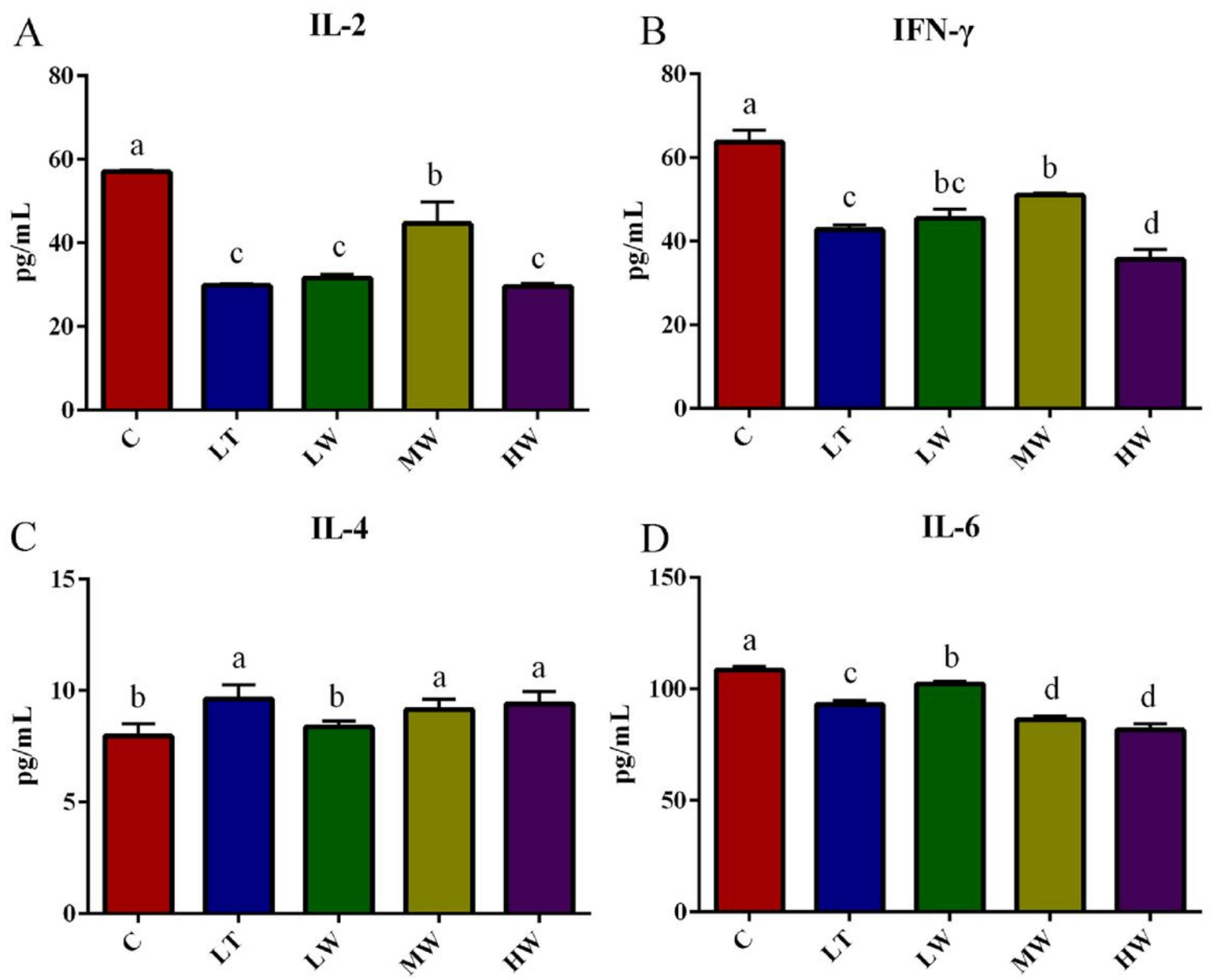

Figure 3

Effect of wind chill on serum levels of cellular inflammatory factors in sheep. (A) IL-2. (B) IL-4. (C) IFN-ץ. (D) IL-6. 


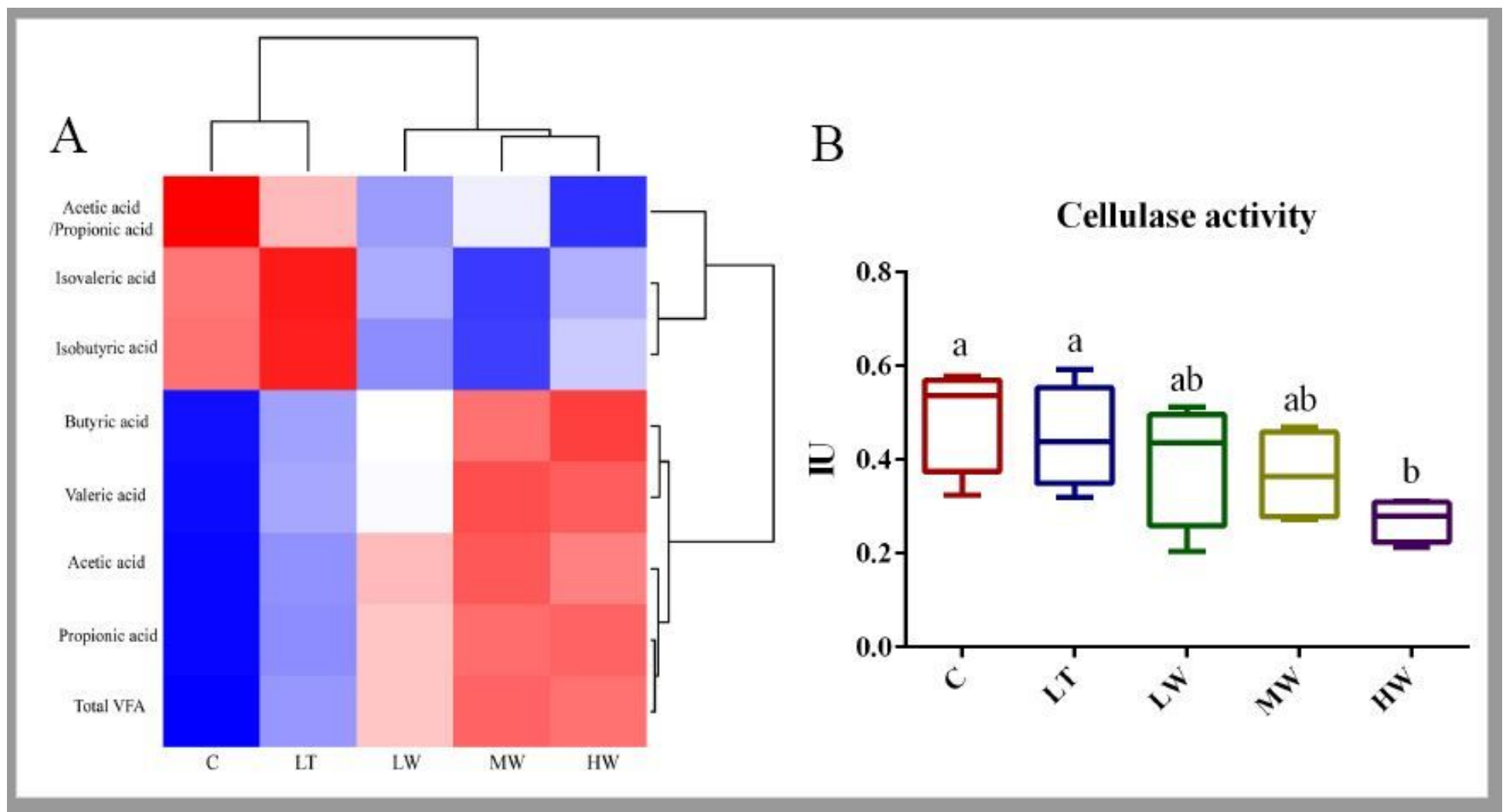

Figure 4

The effects of wind chill on the concentration of VFA and cellulase activity in the rumen. (A) VFA. (B) cellulase activity. Red indicates a high content, and blue indicates a low content. 


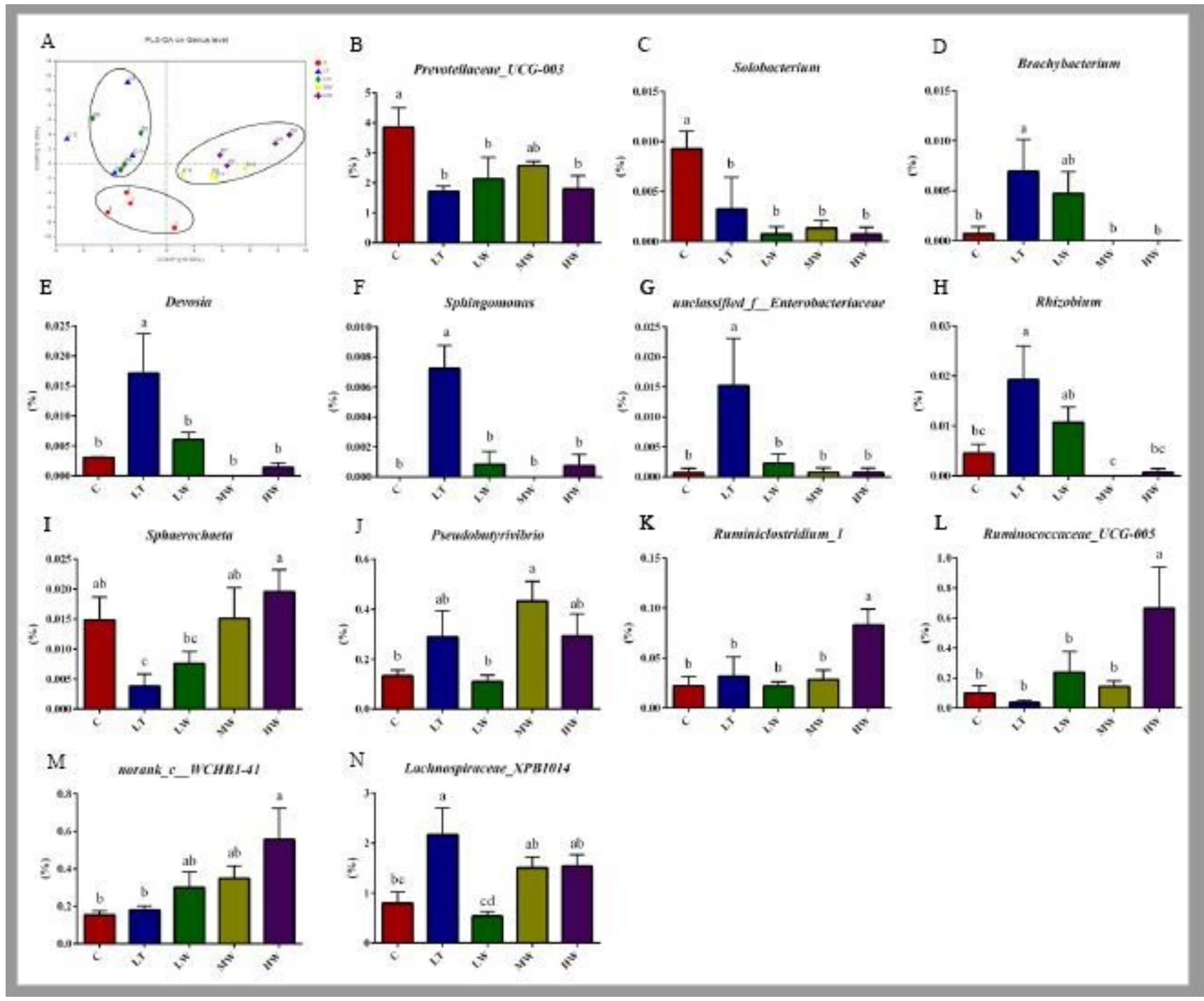

Figure 5

The effects of wind chill on the microbiota in the rumen.(A) Beta diversity as evaluated by PLS-DA. (BN)Microbiota at the genus level were significantly changed. (B)Prevotellaceae_UCG-003, (C) Solobacterium, (D) Brachybacterium, (E) Devosia, (F) Sphingomonas, (G) unclassified_f_Enterobacteriaceae, (H) Rhizobium, (I) Sphaerochaeta, (J) Pseudobutyrivibrio, (K) Ruminiclostridium_1, (L) Ruminococcaceae_UCG-005, (M) norank_c_WCHB1-41, (N) Lachnospiraceae_XPB1014. 

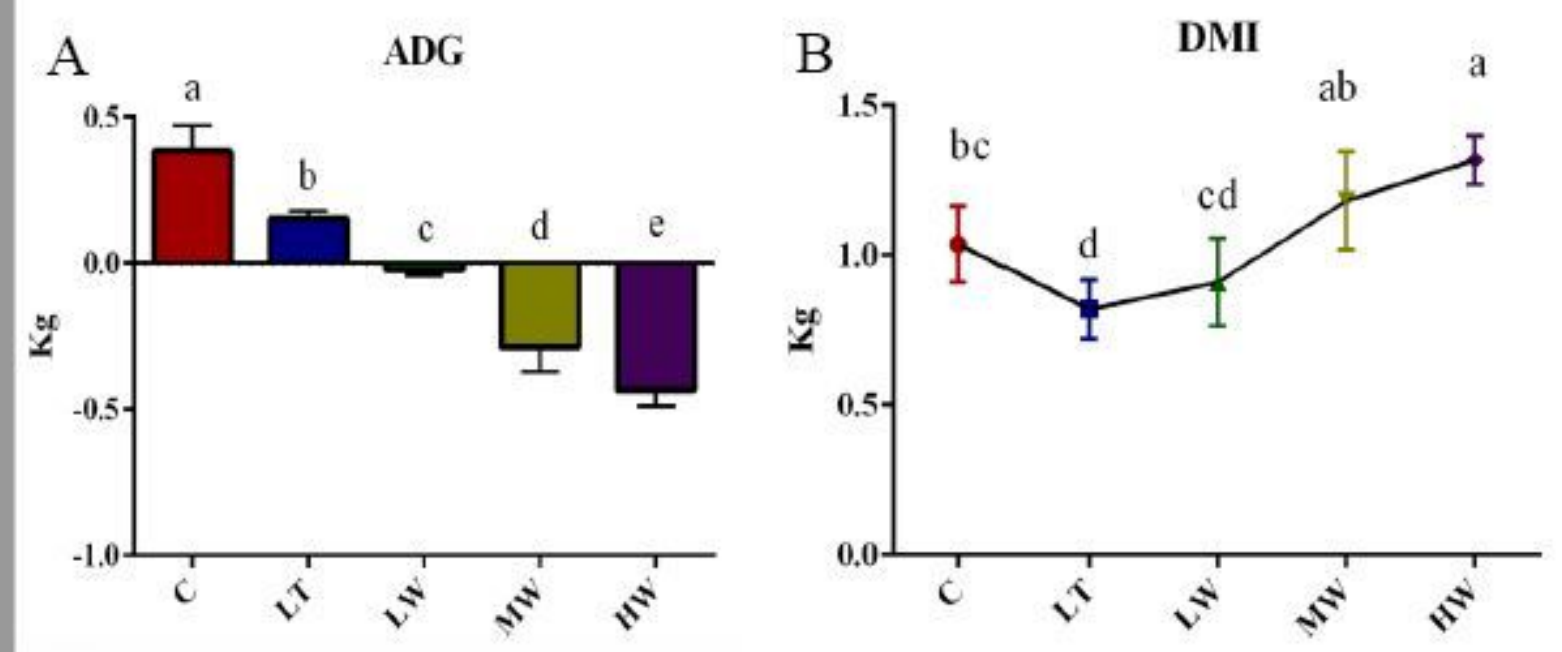

C apparent digestibility of dry matter
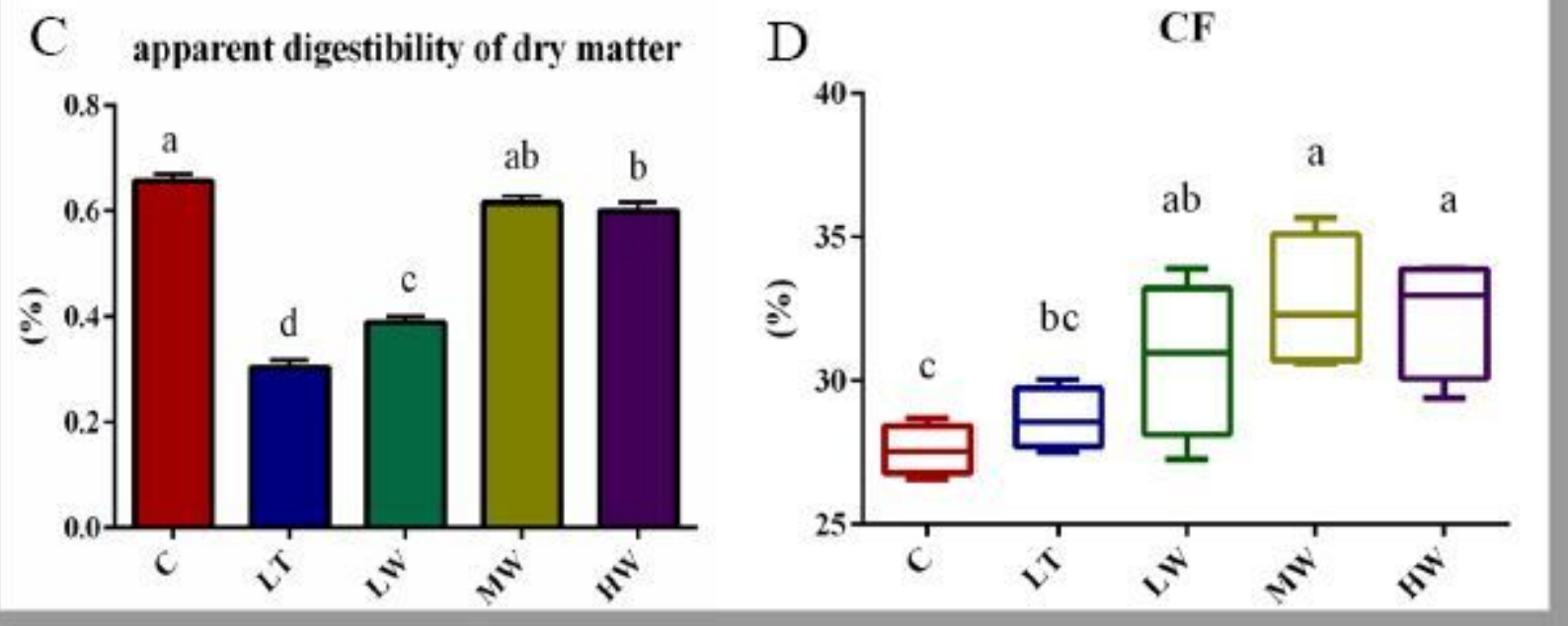

Figure 6

Effect of low temperature on the growth index of sheep.(A) Average daily gain (ADG) changes in sheep.

(B) Dry matter intake (DMI) of each group of sheep. (C) Apparent digestibility of Dry matter (DM).d. Crude fiber (CF) in feces. $\mathrm{C}$ (sheep exposed to $5^{\circ} \mathrm{C}$ ), $\mathrm{LT}$ (sheep exposed to $-15^{\circ} \mathrm{C}$ and an average wind velocity less than $0.5 \mathrm{~m} / \mathrm{s}$ ), LW (sheep exposed to $-15^{\circ} \mathrm{C}$ and an average wind velocity of $3 \mathrm{~m} / \mathrm{s}$ ), MW (sheep exposed to $-15^{\circ} \mathrm{C}$ and an average wind velocity of $4 \mathrm{~m} / \mathrm{s}$ ), $\mathrm{HW}$ (sheep exposed to $-15^{\circ} \mathrm{C}$ and an average wind velocity of $5 \mathrm{~m} / \mathrm{s}$ ).

\section{Supplementary Files}

This is a list of supplementary files associated with this preprint. Click to download.

- Supplementarymaterial.docx

- Supplementaryinformation.docx 